\title{
Microbial cocktails raise bar for $C$. diff. treatments
}

\author{
Seres's success with an industrially made bacterial mix in phase 3 trials against Clostridium difficile infection \\ promises an alternative to fecal microbial transplantation in clinical practice.
}

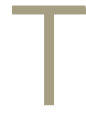
he August announcement of positive data from Seres Therapeutics' phase 3 trial to prevent recurrent Clostridium difficile infections renewed hopes that purified, defined mixtures of bacteria can treat such infections as effectively as and presumably more safely than - fecal microbial transplantation (FMT). Several other companies have now also shown favorable late-stage trial data in ' $C$. diff. using customized stool-derived cocktails. Along with Seres, they are teeing up clinical studies of these compounds in a variety of diseases.

Seres's SER-109 is a mix of bacterial spores of Firmicutes species extracted from the stool of healthy human donors, fractionated and packaged into a capsule. In the phase 3 trial, people in the treatment arm received four capsules per day for three days. After eight weeks, SER-109 reduced the proportion of patients experiencing a recurrence of $C$. diff. infection by $30.2 \%$ versus placebo, thus meeting the study's primary endpoint. On a conference call discussing the results, Mark Wilcox of the University of Leeds called the drug "at least as effective as FMT." According to guidelines, fecal transplant is a viable alternative treatment approach to multiple recurrent C. diff. infection. "I think SER-109 is reasonably comparable to FMT given by capsule," says Elizabeth Hohmann, who runs the core FMT laboratory at Massachusetts General Hospital.

The phase 3 data are a turnaround from Seres's disappointing phase 2 result with SER-109 in 2016, when, much to the field's dismay, it failed to achieve its primary endpoint. The company was left to examine possible reasons for the failure. A subsequent assessment did not identify any manufacturing issues; nonetheless, when embarking on the phase 3 study, Seres increased the drug dose.

"We learned that dose could drive significant differences in kinetics and magnitude of engraftment, which are measures of the pharmacokinetics of the drug," says chief scientist Matthew Henn. "There is a race to repair," he says; increasing the dose approximately tenfold produced a more rapid onboarding of bacteria, helping restructure the microbiome and leading

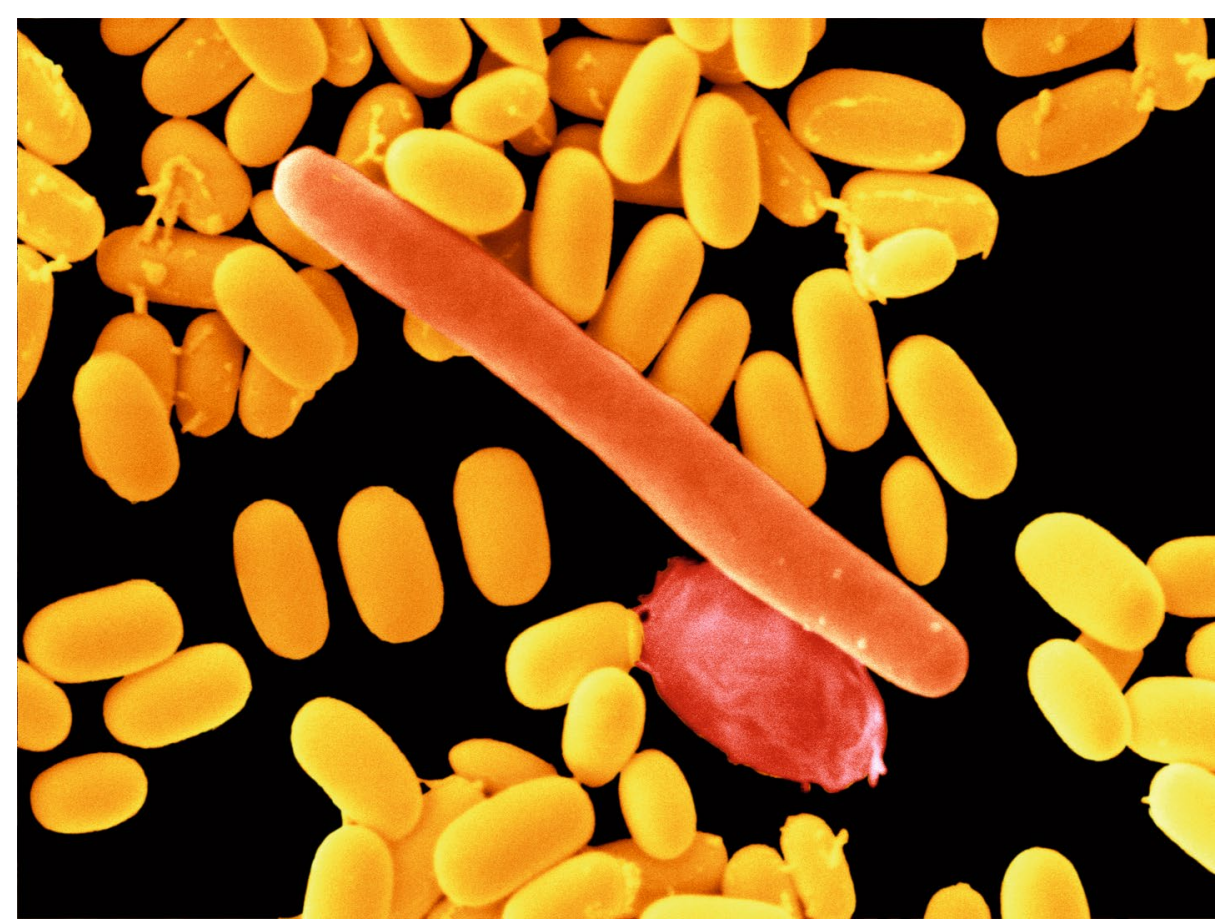

Bacterial mixes extracted from human stool appear to prevent recurrent infections with Clostridium difficile, pictured. Credit: Scimat / Science Source

to generation of microbially associated metabolites that have a meaningful impact on $C$. diff. survival or germination. In the phase 2 , north of $50 \%$ of the infections that recurred did so within one to two weeks after initiation. "It has to happen quickly during the window of vulnerability," he says.

Seres also changed the test it used to identify participants as having C. diff. infection, replacing a commonly used PCR diagnostic with an enzyme-linked immunosorbent assay (ELISA) that, by detecting the toxin the bug produces, can reveal active infection versus mere colonization. Targeting only active infections maximized the chance of separating the drug's effect from placebo, says chief medical officer Lisa von Moltke.

"[Seres] made some important decisions in light of their phase 2 results," says Mark Smith, CEO of Finch Therapeutics, adding that the SER-109 findings are "a beautiful result" and an important outcome for the field. Finch is developing CP101, a stool donation-based treatment in preserved, lyophilized capsules to prevent recurrent C. diff. infections. In a phase 2 study, CP101 demonstrated a $74.5 \%$ sustained response rate over placebo at eight weeks. Finch is readying a large confirmatory phase 3 trial of CP101.

Two other companies, Rebiotix and Vedanta Biosciences, are pursuing microbe-based drugs to stop C. diff infection recurrences, and both are in late-stage development. Rebiotix is running a pivotal phase 3 study of its commercially prepared FMT in enema form. Vedanta Biosciences is expecting a readout of its phase 2 trial of VE303, which, unlike the other three drug candidates, is a defined consortium of eight types of cultured, clonal commensal bacteria strains.

SER-109 is the closest to market, however, and could be approved in the United States in the second half of 2021. Nestlé holds rights outside of North America and has not said whether it will seek an 
approval based on the current data. Nestle also holds ex-US rights to Seres's phase 2 ulcerative colitis candidate SER-287, which follows a manufacturing process similar to SER-109's. Seres also has several early-stage drug candidates comprising bacterial strains grown in culture. The plan is to test them in melanoma patients treated with immunotherapy (functional gut microbiota may influence the outcome from checkpoint inhibitor treatment), to treat colitis and to prevent sepsis from antibiotic-resistant bacteria that could follow hematopoietic stem cell transplantation.

Although stool-derived bacterial mixes appear sufficient to provide the efficacy needed for successful prevention of recurrent C. diff. infection, whether this same onboarding of bacteria translates into success in these other indications is an open question.

It is possible, of course, that the peculiarities of $C$. diff. make the treatment a one-hit wonder. Outside of C. diff., says Karim Dabbagh, CEO of Second Genome, it will be more challenging because other diseases are more complex and multifactorial. C. diff. arises from an absence of microflora after long-term treatment with strong antibiotics. As with FMT, the rationale is that transplanting bacteria restores the microflora and, in so doing, keeps the C. diff. bacteria in check.

"What Seres has done, with a more industrial version of FMT, fits the hypothesis," Dabbagh says. Less clear is whether other diseases now being experimentally treated with bacterial mixes will fit that hypothesis. That's because an individual's microflora tends to be fairly stable over time, notwithstanding changes in diet and environmental conditions.

The links between microflora status and disease modulation certainly exist. Bacteria may take bile acids pumped into the intestine from the liver and turn them into secondary bile acids. The ratio of those primary to secondary bile acids is important: too much of the former and not enough of the latter encourages an environment where $C$. diff., for example, can thrive. $C$. diff. also makes toxins that perforate the barrier, whereas short-chain fatty acids, made by other gut microorganisms breaking down fiber, can help the barrier seal again. Another way to help starve $C$. diff is through competition for nutrients in the intestinal environmental niche and other indirect mechanisms.

The net effect of all those mechanisms on the microbial community goes beyond just helping to fight $C$. diff., says Vedanta CEO Bernat Olle. It also helps reconstitute the entirety of beneficial bacteria. "This has implications outside of $C$. diff. because it is not the only bacteria that takes advantage of dysbiosis that follows antibiotic treatment of high-risk patients in hospitals," he says.

\section{"It is possible, of course, that} the peculiarities of C. diff. make the treatment a one-hit wonder."

\section{Inflammatory diseases like colitis and} infections are next up for the field. MaaT Pharma is going into the clinic with a live microbial therapeutic cocktail, MaaT013, to prevent graft-versus-host disease following bone marrow transplantation. Their approach is based on data showing that FMTs can treat this inflammatory response that can manifest in the gut. Targeting the gut-brain axis is also attractive: Finch's FIN-211, for example, could enter the clinic next year in autism spectrum disorder (ASD), a setting where FMT has shown some promise. FIN-211 will comprise a donor-derived stool-based scaffold with an additional bacterial strain. "There are syntrophies and cross-feeding that goes on," Smith says, whereas other strains (which Finch has not yet identified) have activity, he says, particularly in autism-associated constipation.

The conceit of using fecal bacteria as a scaffold is similar to the approach in tissue engineering, where scientists build tissue scaffolds that hold and deliver growth factors and recruit other growth factors to the target site. Seres has shown it can use donor stool in this way, treat it with ethanol to kill viruses and vegetative bacteria everything except spores from Firmicutes bacteria, which include Clostridia. That small fraction of organisms appears adequate to ward off $C$. diff. In a sense, it is tantamount to having a scaffold through which the other bacteria come back and rebloom after treatment with antibiotics.

FMT promises to remain a valuable research tool to help understand where to expand the field of microbe-based drugs. Biotechs and academic institutions are collaborating. Vedanta is working with Leiden University Medical Center in the Netherlands studying the use of consecutive FMTs to treat non-alcoholic fatty liver disease. Second Genome is actively developing a microbe-based approach to treating ASD, trading on studies showing that if you can reset the microflora in teenagers with ASD by treating with antibiotics and then giving them FMT, it can restore more gastrointestinal functionality and have an effect on the behavioral aspects of autism. To that end, it is collaborating with the Stanford University School of Medicine and Oregon State University via a Phase II Small Business Innovation Research grant to develop a platform for discovery and validation of key metabolites produced by microbes in central nervous system disorders, ASD in particular.

A combination of safety alerts and daunting screening requirements due to SARS-CoV-2 has at the moment sharply curtailed broad use of FMT. By the time the SARS-CoV-2 situation eases, and with SER109 anticipated on the market next year, the economics of treating C. diff. are likely to change. Seres has not commented on pricing of SER-109, but Cowen senior research analyst Joseph Thome is among those pegging it north of $\$ 20,000$ on the basis of the current cost of treatment, including hospitalization.

Fecal transplants are much cheaper. "The stool bank model we have been operating under is doing a really good job at a reasonable cost," says FMT specialist Colleen Kelly, associate professor, Alpert Medical School, Brown University. But the safety checks needed for FMT could be an issue, and an approval for Seres could prompt regulators to limit the activities of stool banks. Insurers, already reticent to cover FMT, could be even less likely to do so.

"I think the upshot will be a lot of pressure to move from true FMT to a regulated product," says Benjamin Mullish of Imperial College London.

FMT is widespread in Italy, the Netherlands and the United Kingdom, says Giovanni Cammarota of the Catholic University of the Sacred Heart in Milan. In other countries, including Germany, France and Spain, it is struggling to take off, mainly because regulators tend to discourage the approach. Nor have the microbiome companies yet established a strong clinical trials presence in Europe, Mullish adds.

\section{Mark Ratner}

Tequesta, FL, USA

Published online: 3 December 2020 https://doi.org/10.1038/s41587-020-00765-8 